\title{
Area Under the Curve from T1 to T2
}

National Cancer Institute

\section{Source}

National Cancer Institute. Area Under the Curve from T1 to T2. NCI Thesaurus. Code C85566.

The area under the curve (AUC) from T1 to T2. 